\title{
SOBREVIVÊNCIA E CRESCIMENTO DE MUDAS DE PESSEGUEIRO INTERENXERTADAS ${ }^{1}$
}

\author{
CHARLES ALLAN TELLES ${ }^{2}$, LUIZ ANTONIO BIASI ${ }^{2}$, UBIRAJARA RIBEIRO MINDÊLLO NETO ${ }^{3}$, \\ EDUARDO PETERS ${ }^{4}$
}

\begin{abstract}
RESUMO - Os experimentos foram realizados no viveiro da Embrapa - Transferência de Tecnologia em Canoinhas -SC, no período de dezembro de 2003 a julho de 2005. Estudou-se o efeito de interenxertos, na produção de mudas de pessegueiro. Os tratamentos foram: combinação de dois porta-enxertos ('Okinawa' e 'Capdeboscq'), quatro interenxertos (ameixeira 'Reubennel' e 'Irati', um umezeiro e uma cerejeira 'Capulin') e duas copas ('Coral' e 'Chimarrita'). O delineamento experimental inicial foi inteiramente ao acaso, com 20 tratamentos, 6 repetições e 8 plantas por parcela. Após a cicatrização e o crescimento dos enxertos, as mudas foram transplantadas para pomar definitivo, sem os tratamentos com os interenxertos de umezeiro e cerejeira que apresentaram baixa sobrevivência com os porta-enxertos de pessegueiro utilizados neste trabalho. $\mathrm{O}$ delineamento neste caso foi em blocos ao acaso, com 12 tratamentos, 4 repetições e 11 plantas por parcela. O crescimento das copas foi avaliado pelas seguintes variáveis: diâmetro $5 \mathrm{~cm}$ acima do ponto de enxertia, comprimento da ramificação principal e número de ramificações secundárias. Com relação ao crescimento das copas, verificou-se que as mudas interenxertadas com ameixeira apresentam um crescimento reduzido, com possibilidade de utilização em pomares mais adensados.
\end{abstract}

Termos para indexação: Prunus persica (L.) Batsch, enxertia, interenxertos, vigor da copa.

\section{SURVIVAL AND GROWTH OF PEACH SEEDLINGS INTERGRAFTING}

\begin{abstract}
The trial was carried through in the fishery of the Embrapa- Technology Transfer in Canoinhas - SC, the period of December of 2003 the July of 2005. The effect of interstock was studied, in the production of peach tree seedlings. The treatments had been the combination of two rootstock ('Okinawa' and 'Capdeboscq'), four interstock (plum tree 'Reubennel' and 'Irati', Umezeiro and cherry 'Capulin') and two scion ('Coral' and 'Chimarrita'). O initial experimental design used were entirely randomized, with 20 treatments, 6 replications and 8 plants for parcel. After the cicatrisation and growth of the scion, the seedlings had been transplanted for definitive orchard, without the treatments with the interstocks of Umezeiro and Cherry that presented low survival with the peach tree rootstocks used in this work. O delineation in this in case that he were block-type randomized with 12 treatments, 4 repetitions and 11 plants for parcel. The growth of the scion was estimated for the variables: diameter $5 \mathrm{~cm}$ above of the point of graft, length of the main ramification and number of secondary ramifications. With relation to the growth of the scion, was verified more that the intergrafting seedlings with plum tree present a reduced growth, with possibility of use in accumulated orchards.
\end{abstract}

Index terms: Prunus persica (L.) Batsch, grafting, interstock, treetop vigor.

\section{INTRODUÇÃO}

Atualmente, a fruticultura moderna vem buscando novas técnicas para a redução do porte das árvores e a obtenção de plantas compactas e com produtividade equivalente. A inserção de um genótipo diferente entre a copa e o porta-enxerto pode ser utilizada na tentativa de diminuição do vigor das plantas e na melhoria da compatibilidade entre as partes enxertadas.

A interenxertia que, segundo Hartmann et al. (1990), consiste na utilização de um fragmento de caule intermediário ou filtro compatível entre o porta-enxerto e o enxerto, pode influenciar no desenvolvimento da copa e das raízes. Esta é uma técnica que, em regra, tem o objetivo de diminuir o vigor das plantas, aumentar a eficiência produtiva e melhorar a qualidade das frutas, conforme já verificado em diversas espécies frutíferas, como cerejeira (Larsen et al., 1987; Rozpara et al., 1990), macieira (Koike \& Tsukahara, 1988), pereira (Westwood et al., 1989), damasqueiro (Ogasanovic et al., 1991) e ameixeira (Grzyb et al., 1994).

Porém, Reighard (1995) destaca que o uso de interenxertia não tem sido comumente aplicado na América do Norte, na produção de mudas de pessegueiro, devido aos resultados de certo modo contraditórios, ao maior custo de produção da muda e à fraca união entre as partes enxertadas. Mas, apesar disto, o mesmo autor observou uma melhora significativa na produção em função da área da seção do tronco (eficiência produtiva) quando da utilização da interenxertia. Entretanto a produção e a qualidade dos frutos observados entre plantas, com interenxerto ou não, foram similares. O mesmo autor também observou que os interenxertos de pessegueiro 'Ta Tao 4' e 'Ta Tao 24' retardaram o florescimento de oito cultivares de pessegueiros, evitando, com isso, os danos causados por geadas tardias, durante ou imediatamente após a floração, contrariando Scarpare Filho et al. (2000) que, de maneira geral, observaram que a presença do interenxerto da ameixeira 'Januária' antecipou a floração e a brotação das cultivares de pessegueiro Tropical e Ouromel-2, conseguindo uma colheita antecipada, demonstrando que cada cultivar ou espécie tem um comportamento diferente.

Interenxertos com ameixeiras têm sido testados por alguns autores para superar a incompatibilidade entre copas e porta-enxertos não comerciais de pessegueiro e na tentativa de controle no tamanho das copas e melhoria da produção. Entretanto, poucos resultados satisfatórios têm sido alcançados.

Este trabalho teve como objetivo estudar o efeito de interenxertos na produção de mudas de pessegueiro, verificando a sobrevivência dos materiais enxertados e o desenvolvimento vegetativo das mudas.

\section{MATERIAL E MÉTODOS}

Os experimentos foram conduzidos em 2 etapas (enxertia e verificação do crescimento), na Estação Experimental da Embrapa (Empresa Brasileira de Pesquisa Agropecuária) - Transferência de Tecnologia, localizado na cidade de Canoinhas-Santa Catarina, com

\footnotetext{
${ }^{1}$ (Trabalho 173-2005). Recebido: 19-10-2005. Aceito para publicação: 10-05-2006.

${ }^{2}$ Eng. ${ }^{\circ}$ Agrônomo, Aluno do Programa de Pós-Graduação em Agronomia, Produção Vegetal. UFPR. Bolsista da CAPES. e-mail: charles.allan@bol.com.br.

${ }^{2}$ Professor, Dr. Adjunto do Departamento de Fitotecnia e Fitossanitarismo. Setor de Ciências Agrárias. UFPR. Caixa Postal 19.061. CEP 81531-990. CuritibaPR. Fone: (41) 3350-5601. e-mail: biasi@ufpr.br. Bolsista de Produtividade em Pesquisa do CNPq.

${ }^{3}$ Eng. ${ }^{\circ}$ Agrônomo, M.Sc. Embrapa Transferência de Tecnologia/SNT Canoinhas-SC. e-mail: ubirajara.encan @embrapa.br.

${ }^{4}$ Técnico Agropecuário. Embrapa Transferência de Tecnologia/SNT. Canoinhas-SC.
} 
clima do tipo $\mathrm{Cfb}$, segundo Köppen: temperado constantemente úmido, sem estação seca definida e com verão fresco, no período de dezembro de 2003 a julho de 2005.

\section{Enxertia dos filtros, das copas e sua condução.}

Entre os dias $1^{\circ}$ e 3 de dezembro de 2003, quando os portaenxertos das cultivares Okinawa e Capdeboscq, obtidos a partir de sementes, se encontravam com um ano e meio de idade, foram enxertadas borbulhas das cultivares Irati e Reubennel de ameixeira (Prunus salicina), de umezeiro (Prunus mume Sieb et Zucc) e cerejeira cultivar Capulin (Prunus serotina) para serem utilizados como interenxertos. Foi realizada enxertia de gema ativa, pelo método de borbulhia em "T" invertido, a $20 \mathrm{~cm}$ do solo. As mudas foram conduzidas com uma só brotação, sendo tutoradas para um crescimento mais vertical e reto, sem ramos ladrões ou brotações do porta-enxerto.

Já a enxertia das duas cultivares-copa de pessegueiro (Prunus persica (L.) Batsch), 'Chimarrita' e 'Coral', foi realizada dos dias 21 a 23 de junho de 2004, por garfagem em inglês complicado no campo. Os filtros (interenxertos), nessa ocasião, apresentavam um diâmetro variando de 4 a $12 \mathrm{~mm}$. Os filtros foram padronizados com uma altura de $15 \mathrm{~cm}$. Os ramos das copas que foram enxertados apresentavam em média $10 \mathrm{~cm}$ de comprimento, com presença de duas gemas.

As mudas foram conduzidas em viveiro, com espaçamento de 1,20 x 0,25 metros, com vistorias periódicas e com pulverizações de fungicidas e inseticidas, conforme o aparecimento de problemas fitossanitários, até serem transferidas para área de pomar definitivo.

Os tratamentos foram: 'Capdeboscq' + ameixa 'Reubennel' + pêssego 'Coral'; 'Capdeboscq' + ameixa 'Reubennel' + pêssego 'Chimarrita'; 'Capdeboscq' + ameixa 'Irati' + pêssego 'Coral'; 'Capdeboscq' + ameixa 'Irati' + pêssego 'Chimarrita'; 'Capdeboscq' + cereja 'Capulin' + pêssego 'Coral'; 'Capdeboscq' + cereja 'Capulin' + pêssego 'Chimarrita'; 'Capdeboscq' + umezeiro + pêssego 'Coral'; 'Capdeboscq' + umezeiro + pêssego 'Chimarrita'; 'Okinawa' + ameixa 'Reubennel' + pêssego 'Coral'; 'Okinawa' + ameixa 'Reubennel' + pêssego 'Chimarrita'; 'Okinawa' + ameixa 'Irati' + pêssego 'Coral'; 'Okinawa' + ameixa 'Irati' + pêssego 'Chimarrita'; 'Okinawa' + cereja 'Capulin' + pêssego 'Coral'; 'Okinawa' + cereja 'Capulin' + pêssego 'Chimarrita'; 'Okinawa' + umezeiro + pêssego 'Coral'; 'Okinawa' + umezeiro + pêssego 'Chimarrita'; 'Capdeboscq' + pêssego 'Coral' (muda sem interenxerto); 'Capdeboscq' + pêssego 'Chimarrita' (muda sem interenxerto); 'Okinawa' + pêssego 'Coral' (muda sem interenxerto), e 'Okinawa' + pêssego 'Chimarrita' (muda sem interenxerto).

$\mathrm{O}$ delineamento experimental utilizado foi inteiramente ao acaso, com 20 tratamentos, 6 repetições e 8 plantas por parcela.

Após 51 dias da enxertia, foi avaliada a sobrevivência dos enxertos (filtros), contabilizada pela porcentagem de gemas brotadas e gemas mortas (oxidadas).

Após 135 dias da enxertia dos filtros, foi realizada uma avaliação no crescimento dos interenxertos, observando-se as seguintes variáveis: comprimento do broto $(\mathrm{cm})$, diâmetro a $5 \mathrm{~cm}$ acima do ponto de enxertia $(\mathrm{mm})$ e número de ramificações secundárias por broto.

Essas mudas foram ainda avaliadas no momento da retirada do viveiro e transplante para o pomar definitivo, verificando a sobrevivência da enxertia das copas.

\section{Verificação a campo do desenvolvimento da plantas}

Nos dias 11 e 12 de agosto de 2004 (50 dias após a enxertia das copas), as mudas foram transplantadas com raiz nua para área de pomar definitivo, com espaçamento de 4 x $3 \mathrm{~m}$.

Os tratamentos no pomar foram aqueles onde a enxertia se mostrou compatível, sendo: 'Capdeboscq' + ameixa 'Reubennel' + pêssego 'Coral'; 'Capdeboscq' + ameixa 'Reubennel' + pêssego 'Chimarrita'; 'Capdeboscq' + ameixa 'Irati' + pêssego 'Coral';
'Capdeboscq' + ameixa 'Irati' + pêssego 'Chimarrita'; 'Okinawa' + ameixa 'Reubennel' + pêssego 'Coral'; 'Okinawa' + ameixa 'Reubennel' + pêssego 'Chimarrita'; 'Okinawa' + ameixa 'Irati' + pêssego 'Coral'; 'Okinawa' + ameixa 'Irati' + pêssego 'Chimarrita'; 'Capdeboscq' + pêssego 'Coral' (muda sem interenxerto); 'Capdeboscq' + pêssego 'Chimarrita' (muda sem interenxerto); 'Okinawa' + pêssego 'Coral' (muda sem interenxerto), e 'Okinawa' + pêssego 'Chimarrita' (muda sem interenxerto)

O delineamento experimental utilizado nesse caso foi em blocos ao acaso, com 4 repetições e 11 plantas por parcela.

Em junho de 2005, foi realizada uma análise de crescimento das mudas, verificando-se as seguintes variáveis: tamanho das brotações emitidas $(\mathrm{cm})$, número de ramificações presentes nessa brotação e diâmetro dos brotos $5 \mathrm{~cm}$ acima do ponto de enxertia.

Os resultados obtidos foram submetidos ao teste de Bartllet para testar a homogeneidade entre as variâncias dos tratamentos e, depois, submetidos à análise de variância para verificar se houve diferença entre os tratamentos testados. Nos casos onde o teste $\mathrm{F}$ foi significativo, as médias dos tratamentos foram analisadas pelos testes de Tukey, a 1 e $5 \%$ de probabilidade, utilizando-se do programa estatístico MSTATC.

\section{RESULTADOS E DISCUSSÃO}

A porcentagem de sobrevivência da enxertia dos filtros foi perfeita para as ameixeiras, obtendo-se uma taxa de $100 \%$ para ambos os porta-enxertos (Tabela 1), sendo superior estatisticamente ao umezeiro, que obteve uma taxa média de $19,3 \%$, enquanto para 'Capulin' a taxa média (considerando os dois porta-enxertos) foi de $2,08 \%$. Esses resultados mostram que as ameixeiras apresentam uma alta taxa de sobrevivência, devida a sua proximidade genética e similaridade morfológica, desenvolvendo uma proliferação de células meristemáticas e parenquemáticas com afinidades para a formação do novo sistema de condução (floema e xilema) e na lignificação da parede celular. Hartmann et al. (1990) e Errea (1998) citam que, para haver uma boa e sólida união entre as partes enxertadas, deve existir uma proliferação de células parenquimáticas, ocorrendo a formação de um tecido caloso na região cambial, com posterior diferenciação destas células, formando um novo câmbio e uma nova conecção vascular, permitindo assim o sucesso da enxertia.

TABELA 1 - Porcentagem de gemas brotadas e gemas mortas dos interenxertos de ameixeiras 'Reubennel' e 'Irati', umezeiro e cerejeira cv Capulin, após 51 dias da borbulhia nos porta-enxertos 'Capdeboscq' e 'Okinawa', de 1,5 ano de idade. Canoinhas-SC, 2003.

\begin{tabular}{|c|c|c|c|c|}
\hline \multirow[b]{2}{*}{ Interenxertos } & \multicolumn{2}{|c|}{ Gemas brotadas (\%) } & \multicolumn{2}{|c|}{ Gemas mortas (\%) } \\
\hline & Capdeboscq & Okinawa & Capdeboscq & Okinawa \\
\hline Irati & $100 \mathrm{aA}$ & 100 a $\mathrm{A}$ & $0 \mathrm{c} \mathrm{A}$ & $0 \mathrm{c} \mathrm{A}$ \\
\hline Reubennel & 100 a A & 100 & $0 \mathrm{c} \mathrm{A}$ & $0 \mathrm{c} \mathrm{A}$ \\
\hline Umezeiro & $30,2 \mathrm{~b} \mathrm{~A}$ & $8,3 \mathrm{~b}$ B & 69,8 b B & $91,7 \mathrm{~b} \mathrm{~A}$ \\
\hline Capulin & $3,1 \mathrm{c} \mathrm{A}$ & $1,04 \mathrm{c} \mathrm{B}$ & 96,9 а $\mathrm{B}$ & 98,96 a A \\
\hline CV (\%) & \multicolumn{2}{|c|}{$\mathbf{1 , 0 7}$} & \multicolumn{2}{|l|}{ o, } \\
\hline
\end{tabular}

1 Médias seguidas de mesma letra maiúscula na linha e minúscula na coluna não diferem significativamente entre si, ao nível de $5 \%$ de probabilidade, pelo teste de Tukey.

Essa combinação celular pode estar relacionada com moléculas de proteínas no plasmalema, as quais se movimentam entre as células, formando um complexo com atividade catalítica e, uma vez não formado este complexo, pode ocorrer uma rejeição mútua de células de condução, gerando uma baixa sobrevivência de enxertia (Yeoman \& Brown, 1976).

Observando a porcentagem de gemas mortas, verificou-se uma grande rejeição nas enxertias realizadas com 'Capulin', sendo superior aos demais, com porcentagens de mortalidade acima de $96 \%$, 
TABELA 2 - Crescimento das brotações dos diferentes interenxertos 'Irati', 'Reubennel', Umezeiro e 'Capulin', após 135 dias da enxertia, em dezembro de 2003, pela técnica de borbulhia, sobre dois porta-enxertos de pessegueiro 'Capdeboscq' e 'Okinawa', Canoinhas-SC.

\begin{tabular}{ccccccc}
\hline Interenxertos & \multicolumn{2}{c}{ Comprimento médio das brotações $(\mathrm{cm})$} & Diâmetro médio das brotações $(\mathrm{cm})$ & \multicolumn{2}{c}{ Número de ramificações nas brotações } \\
\cline { 2 - 7 } & Capdeboscq & Okinawa & Capdeboscq & Okinawa & Capdeboscq & Okinawa \\
\hline Irati & $84,1 \mathrm{a}$ & $86,3 \mathrm{a}$ & $0,57 \mathrm{a}$ & $0,6 \mathrm{a}$ & $3,0 \mathrm{a}{ }^{\text {ns }}$ & $3,4 \mathrm{a}$ \\
Reubennel & $76,2 \mathrm{a}$ & $81,6 \mathrm{a}$ & $0,54 \mathrm{a}$ & $0,52 \mathrm{a}$ & $2,1 \mathrm{~b}$ & $1,6 \mathrm{~b}$ \\
Umezeiro & $31,6 \mathrm{~b}$ & $30,8 \mathrm{~b}$ & $0,18 \mathrm{~b}$ & $0,18 \mathrm{~b}$ & $1,6 \mathrm{~b}$ & $0,8 \mathrm{~b}$ \\
Capulin & $0 \mathrm{c}$ & $0 \mathrm{c}$ & $0 \mathrm{c}$ & $0 \mathrm{c}$ & $0 \mathrm{c}$ & $0 \mathrm{c}$ \\
\hline CV (\%) & \multicolumn{7}{c}{$\mathbf{1 7 , 7 9}$} & & $\mathbf{1 9 , 4 7}$ & & $\mathbf{3 6 , 3 5}$
\end{tabular}

Médias seguidas de mesma letra minúscula, na coluna, não diferem significativamente entre si, ao nível de 5\% de probabilidade, pelo teste de Tukey.

Médias comparadas nas linhas não foram significativas (ns).

TABELA 3 - Crescimento das copas de pessegueiro 'Coral' e 'Chimarrita', um ano após a enxertia pelo método de garfagem lenhosa, em julho de 2004, sobre os interenxertos 'Irati' e 'Reubennel' e porta-enxertos 'Capdeboscq' e 'Okinawa', Canoinhas-SC.

\begin{tabular}{|c|c|c|c|}
\hline Interenxerto & $\begin{array}{c}\text { Diâmetro do tronco a } 5 \mathrm{~cm} \text { do ponto } \\
\text { de enxertia }(\mathrm{cm})\end{array}$ & $\begin{array}{l}\text { Comprimento da brotação } \\
\text { principal da copa }(\mathrm{cm})\end{array}$ & $\begin{array}{c}\text { Número de ramificações } \\
\text { secundárias da copa }\end{array}$ \\
\hline Sem Interenxerto & $0,906 \mathrm{a}$ & $43,77 \mathrm{a}$ & $6,28 \mathrm{a}$ \\
\hline Irati & $0,665 \mathrm{~b}$ & $26,95 \mathrm{~b}$ & $2,37 \mathrm{~b}$ \\
\hline Reubennel & $0,616 \mathrm{~b}$ & $26,74 \mathrm{~b}$ & $2,16 \mathrm{~b}$ \\
\hline \multicolumn{4}{|l|}{ Porta-enxertos } \\
\hline Capdeboscq & $0,72^{\mathrm{ns}}$ & $31,44^{\mathrm{ns}}$ & $3,05^{\mathrm{ns}}$ \\
\hline Okinawa & 0,74 & 33,53 & 4,15 \\
\hline \multicolumn{4}{|l|}{ Copas } \\
\hline Chimarrita & $0,73^{\mathrm{ns}}$ & $34,58^{\mathrm{ns}}$ & $3,81^{\mathrm{ns}}$ \\
\hline Coral & 0,73 & 30,40 & 3,40 \\
\hline CV (\%) & 17,11 & 18,25 & 36,93 \\
\hline
\end{tabular}

Médias seguidas de mesma letra minúscula, na coluna, não diferem significativamente entre si, ao nível de $1 \%$ de probabilidade, pelo teste de Tukey.

seguidas da enxertia com umezeiro, que apresentaram uma taxa média de mortalidade de $80 \%$, mostrando que estes materiais não foram compatíveis com os porta-enxertos testados. A taxa de mortalidade foi um pouco menos acentuada com a cultivar Capdeboscq, chegando a obter $30,2 \%$ de sobrevivência dos enxertos com o umezeiro. Porém esses resultados não são satisfatórios para a produção de mudas, pois, segundo Ojima et al. (1977); Campo Dall'Orto et al. (1984) e Raseira \& Medeiros (1998), uma taxa satisfatória de sobrevivência deve ser acima de $84 \%$. A taxa média de sobrevivência das copas de pessegueiro 'Coral' e 'Chimarrita' sobre os interenxertos de ameixeira 'Irati' e 'Reubennel' foi de $98 \%$, sendo assim satisfatória.

Com relação ao crescimento dos interenxertos (comprimento, diâmetro e número de ramificações secundárias da copa), não houve interação significativa entre porta-enxertos e filtros (Tabela 2). Verificou-se que as ameixeiras tiveram um desenvolvimento maior em relação ao umezeiro, e o ‘Capulin' não apresentou crescimento nenhum devido à alta mortalidade. Estes resultados de crescimento menos acentuados do umezeiro estão de acordo com Campo-Dall'Orto et al. (1992), que já utilizaram o umezeiro ou damasqueiro para induzir o nanismo em pessegueiro, pois este apresenta menor vigor e uma tendência de menor crescimento. Outro fator que pode ter influenciado em seu crescimento final, foi a baixa sobrevivência apresentada por esse material (Tabela 1), havendo alta rejeição, o que pode gerar uma baixa translocação de seiva, conseqüentemente menor crescimento.

Já o crescimento das copas apresentou diferença significativa entre mudas interenxertadas e mudas sem interenxerto, não havendo interação significativa entre as combinações (Tabela 3). Constatouse que mudas interenxertadas apresentaram uma redução do vigor da copa, com menor diâmetro do tronco, comprimento e número de ramificações secundárias, quando comparadas com as plantas sem interenxertos, ocorrendo comportamento semelhante nas duas cultivares-copa estudadas. Estes resultados demonstram que o uso de filtro provoca a redução no crescimento e no vigor das plantas, o que concorda com diversos autores (Westwood et al., 1989; Ogasanovic et al., 1991; Reighard, 1992; Grzyb et al., 1994; Scarpare Filho et al., 2000).

De maneira geral, a presença de ameixeira como filtro na produção de mudas de pessegueiro reduziu o crescimento inicial das plantas, tornando as copas mais compactas, podendo, no futuro, promover maior facilidade nos tratos culturais, principalmente nas operações de poda, raleio e colheita, dispensando o uso de escada, concordando com resultados já obtidos por outros autores em pesquisas de adensamento de plantio (Campo-Dall'Orto et al., 1984; Erez, 1985).

Essa redução do crescimento pode ser influenciada por fatores indiretos relacionados com o uso do interenxerto, uma vez que fatores internos, como circulação de água, nutriente e reguladores vegetais, são afetados pelo uso do filtro, provocando interferência no crescimento da planta, no florescimento e na frutificação (Dana et al., 1963; Hartmann et al. 1990). Além do que, o interenxerto altera a distribuição de hormônios, como as giberelinas (Richards et al., 1986), e modifica os teores de nutrientes minerais na copa das plantas, sendo que a redução no teor de potássio pode causar redução no crescimento vegetativo (Rozpara et al., 1990).

Nesse sentido, a interenxertia apresenta potencial de uso na cultura do pessegueiro, podendo ser utilizada para formação de pomares mais compactos, porém, a nível comercial, depende de pesquisas complementares, para verificar o crescimento, a produção e a longevidade das plantas.

\section{CONCLUSÕES}

1) Não se recomenda enxertar umezeiro e cerejeira 'Capulin' sobre porta-enxertos de pessegueiro, devido a sua baixa taxa de sobrevivência.

2) O uso de ameixeiras 'Irati' e 'Reubennel' como filtro, na produção de mudas de pessegueiro, mostrou-se compatível e induziu a redução do crescimento das copas.

\section{AGRADECIMENTOS}

Os autores agradecem à Embrapa Transferência de Tecnologia, Canoinhas-SC, pelo apoio para a realização desta pesquisa, e ao Dr. José Francisco Pereira da Embrapa Clima Temperado, Pelotas-RS, pelo fornecimento das borbulhas de umezeiro e cerejeira 'Capulin' utilizadas na enxertia. 


\section{REFERÊNCIAS}

CAMPO-DALL'ORTO, F.A.; OJIMA, M.; BARBOSA, W.; MARTINS, F.P. O nanismo do pessegueiro induzido pela enxertia no damasqueiro-japonês. Pesquisa Agropecuária Brasileira, Brasília, v.27, n.3, p.517-521, 1992.

CAMPO-DALL'ORTO, F.A.; OJIMA, M.; BARBOSA, W.; TOMBOLATO, A.F.C.; RIGITANO, O.; ALVES, S. Cultivo de seleções de pessegueiros precoces no sistema de pomar compacto com poda drástica. Pesquisa Agropecuária Brasileira, Brasília, v. 19, n.6, p.719-727, 1984.

DANA, M.M.; LANTZ, H.L.; LOOMIS,W.E. Studies in translocation acroos dwarf interstocks. American Society for Horticultural Science Proceedings, Alexandria, v.82, n.1, p.16-24, 1963.

EREZ, A. Peach meadow orchard. Acta Horticulturae, Wageningen, n.173, p.405-411, 1985.

ERREA, P. Implications of phenolic compounds in graft incompatibility in fruit tree species. Scientia Horticulturae, Zaragoza, v.74, p.195205, 1998.

GRZYB, Z.S.; ROZPARA, E.; HARTMANN, W. The influence of different interstems on growth and yield of plum cv. Ruth Gerstetter trees. Acta Horticulturae, Wageningen, n.359, p.256259, 1994.

HARTMANN, N.T.; KESTER, D.E.; DAVIES JUNIOR, F.T. Plant propagation: principles and practices. $5^{\text {th }}$ ed. Englewood Cliffs: Regents/Prentice-Hall, 1990. 647p.

KOIKE, H.; TSUKAHARA, K. Various interstem effects in combination with 'Marubakaido N-1' rootstock on 'Fuji' apple growth. HortScience, Alexandria, v.23, n.3, p.580-581, 1988.

LARSEN, F.E.; HIGGINS, S.S.; FRITTS JUNIOR, R. Scion/interstock/ rootstock effect on sweet cherry yield, tree size and yield efficiency. Scientia Horticulturae, Amsterdam, v.33, n.3/4, p.237247, 1987.
OGASANOVIC, D.; PLAZINIC, R.M.; PAPIC, V.M. Results from the study of some early apricot cultivars on various interstocks. Acta Horticulturae, Wageningen, n.193, p.383-389, 1991.

OJIMA, M.; CAMPO-DALL'ORTO, F.A.; RIGITANO, O. Obtenção precoce de mudas de pessegueiro. Campinas: Instituto Agronômico, 1977. 13p (Boletim Técnico, 45).

RASEIRA,M.C.B.; MEDEIROS,C.A.B. A cultura do pessegueiro. Brasília: Embrapa-SPI; Pelotas: Embrapa-CPACT,1998. 351p

REIGHARD, G.L. Using interstems to delay bloom in peach. Compact Fruit Tree, London, v.25, n.1, p.90-91, 1992.

REIGHARD, G.L. Use of peach interstem to delay peach phenology. Acta Horticulturae, Wageningen, n. 395, p.201-207, 1995.

RICHARDS, D.; THOMPSON, W.K.; PHARIS, R.P. The influence of dwarfing interstocks on the distribution and metabolism of xylemapplies, gibberellin $\mathrm{A}_{4}$ in apple. Plant Physiology, Rockville, v.82, n.7, p.1090-1095, 1986.

ROZPARA, E.; GRZYB, Z.S.; OLSZEWSKI, T. The mineral content in leaves of two sweet cherry cvs with interstem. Acta Horticulturae, Wageningen, n.274, p.405-412, 1990.

SCARPARE FILHO, J.A.; KLUGE, R.A.; VICTÓRIA FILHO, R.; TESSARIOLINETO, J.; JACOMINO A.P. Comportamento de duas cultivares de pessegueiro com interenxerto da ameixeira 'Januária'. Pesquisa Agropecuária Brasileira, Brasília, v.35, n.4, p.757-765; 2000.

YEOMAN, M.M.; BROWN, R. Implications of the formation of the graft union for organisation in the intact plant. Annual Botanic, London, v.40, p.1265-1276, 1976.

WESTWOOD, M.N.; LOMBARD, P.B.; BJORNSTAD, H.O. Pear on 'Winter Banana' interstem with M.26 apple rootstock. HortScience, Alexandria, v.24, n.5, p.765-767, 1989. 